\title{
Estudio numismático de monedas bajoimperiales procedentes del yacimiento subacuático de Lavaculos (Sancti Petri. Cádiz)
}

María Dolores López de la Orden Facultad de Filosofía y Letras Universidad de Cádiz

Mercedes Gallardo Abárzuza Centro de Arqueología Subacuática del IAPH

\section{Francisco José Blanco Jiménez} Arqueólogo

\section{Resumen:}

Estudio numismático de un tesorillo de monedas bajoimperiales halladas en el yacimiento arqueológico subacuático de Lavaculos (Sancti-Petri, Cádiz), está formado exclusivamente por monedas de bronce de escaso valor y gran desgaste. El mayor número de monedas corresponde a Teodosio I, seguido por Arcadio, Honorio, Graciano, Valentiniano II, Magno Máximo y Constante.

\section{Palabras claves:}

Arqueología subacuática, Sancti-Petri, tesorillo monedas de bronce, bajoimperio.

En 1994, cuando el equipo que estaba llevando a cabo el Proyecto General de Investigación "Carta Arqueológica Subacuática de la Bahía de Cádiz"l iba a iniciar una nueva campaña, unos buceadores de Protección Civil de San Fernando (Cádiz) comunicaron el hallazgo de una serie de restos arqueológicos en el Caño de Sancti-Petri. El riesgo de expolio que corría el yacimiento hizo aconsejable -de común acuerdo con la Delegación Provincial de Cultura de Cádizefectuar una intervención en esta zona ubicada frente a la playa de Lavaculos, en el interior del Caño de Sancti-Petri, casi en el centro del cauce y cerca de la desembocadura, a una profundidad media de $10 \mathrm{~m}$, 
en un área problemática para el buceo debido a la escasa visibilidad, fuertes corrientes de marea e intenso tráfico marítimo de carácter deportivo.

Se planteó una prospección intensiva con recogida del material de superficie. Una vez elegido un punto $\bigcirc$ -marcado por dos anclas de piedra- se trazaron dos ejes de 50 m cada uno, en los sentidos N-S y E-W, formando cuatro cuadrantes (NE, SE, SW y NW). Sobre éstos se iban montando sectores con un ángulo de $15^{\circ}$, que una vez prospectados se desplazaban hasta barrer toda el área delimitada. Las piezas localizadas se ubicaban tomando la distancia que las separaba del punto $O$ y de uno de los lados del sector, previamente establecido. Mediante este sistema se posicionaron y extrajeron un total de 386 piezas, en su mayoría cerámicas, de las cuales 5 son anclas de piedra y un dedal de bronce. Así mismo, se encontraron diversos elementos constructivos que no se extrajeron.

El buen estado de conservación del material y el haber documentado numerosas piezas enterradas casi en su totalidad -que no fueron extraídas-, indicarían que se trata de un yacimiento in situ y no de una acumulación de restos cerámicos producida por las corrientes.

La cronología se centró en dos bloques diferentes: etapa romana, representada por materiales anfóricos de los siglos I-III d.C., y del IV-V d.C. y etapa medieval con restos cerámicos atribuibles al periodo que va del $\mid X$ a la I ${ }^{a}$ mitad del XII.

Los trabajos realizados no permitieron llevar a cabo una valoración tipológica clara y precisa del yacimiento. Las características de la zona prospectada indujeron a pensar, en un principio, que se trataba de un fondeadero. No obstante, tras un primer análisis del estado de conservación de las piezas y dado que el material cerámico se identificaba con tres momentos culturales muy determinados, parece más preciso considerar el yacimiento como una acumulación de varios pecios de diferentes características y cronologías.

Posteriormente, en los años 1998 y 1999, el Centro de Arqueología Subacuática realizó sendas visitas de inspección con el fin de evaluar la evolución que había seguido el yacimiento ya que la naturaleza del fondo esta determinada por la existencia de dunas móviles que cambian constantemente la fisonomía de la zona. Bajo estas arenas se encuentra en unos casos fango compactado y en otros arena endurecida y erosionada que le da aspecto de roca. En los huecos así formados fue donde se localizaron, en 1998, 54 monedas y 6 en el siguiente año. El estudio de estas 60 monedas es el que se presenta a continuación.

Se trata de un tesorillo de AE2 (sólo hay un AE4) del siglo IV d.C., que, sin lugar a dudas pertenecen al mismo conjunto. La práctica de guardar monedas con el fin de atesorarlas es más frecuente en el Bajo Imperio que en otros momentos de la Antigüedad, debido seguramente a la inseguridad de la época tardorromana. Se suelen atesorar monedas en momentos de crisis, cuan- do hay invasiones o en cualquier situación de inestabilidad política o económica, circunstancias que se dan en este momento histórico. Por ejemplo, las monedas de esta época sufrieron devaluaciones y algunas fueron desapareciendo paulatinamente como los bronces denominados AE2, AE3 y AE4. T. Marot $(1994,94)$ dice que la constante inflación afectó a los nominales de poco valor, desapareciendo el AE2 en el año 395 d.C., el AE3 entre los años 410 y 423, quedando solo el AE4 ó nummus como única denominación.

El tesorillo de Sancti Petri responde a las características de los más abundantes en época tardorromana, es decir, formado exclusivamente por monedas de bronce, de escaso valor y con gran desgaste. Estos tipos de atesoramientos evidencian el protagonismo de la moneda de bronce en ciertos niveles económicos de la sociedad tardorromana (Marot, 1994, 92).

En cuanto al contenido de este tesorillo, se ha encontrado un mayor número de monedas de Teodosio I, en total 22 monedas de este emperador. Le siguen, en cantidad, las de Arcadio, que suman 15; en tercer lugar las pertenecientes al gobierno de Honorio; y en inferior cantidad las de Graciano, cinco; Valentiniano II, tres; Magno Máximo, dos; y Constante, una.

Así pues, se puede decir que se trata de un tesorillo de monedas bajoimperiales, del siglo IV d.C., acuñadas entre los años 337 y 395, fecha ésta última en que se desmonetiza el AE2. Se trata, pues, de un atesoramiento con una cronología no muy amplia, 58 años, lo que inclina a pensar en un tesorillo perdido por una causa repentina. Teniendo en cuenta el contexto arqueológico en el que fue hallado, no sería extraño asegurar que fue perdido por su dueño en un naufragio.

La datación de los tesorillos bajoimperiales, de todas formas, es bastante difícil, pues la perduración del uso de las monedas de bronce acuñadas en el siglo IV, hace que la cronología no la proporcione la moneda más tardía del conjunto, sino el tiempo que esta moneda se ha mantenido en circulación (Marot 1994 , 92). Son muy numerosos los tesoros formados por monedas del siglo IV y, si se desconoce el contexto arqueológico, es casi imposible determinar la cronología, ya que pueden ser ocultados en cualquier momento después de la fecha de emisión de la moneda más tardía del conjunto (Marot, 1994, 102). De todas formas, no es éste el caso, pues sí se conoce el contexto arqueológico en el que fue encontrado, un importante yacimiento submarino donde tuvieron lugar numerosos naufragios, cercano al lugar llamado "Rompetimones", nombre bastante elocuente. No estamos, pues, ante una ocultación del tesoro por parte de su dueño, sino de una pérdida de aquél debido al hundimiento del barco donde viajaba.

En resumen, es un tesorillo tardorromano, con las características de los pertenecientes a esta época, formado por monedas de bronce de escaso valor, mayoritariamente $A E 2$, y que abundan mucho en el sur de la Península Ibérica, y más concretamente en la Bética. 


\section{Catálogo}

\section{$\mathrm{N}^{\circ} \mathrm{I}$}

Anverso: DN THEODOSIVS PF AVG. Busto de Teodosio I diademado, de perfil a la derecha, con coraza. (foto I)

Reverso: GLORIA ROMANORVM. Teodosio de pie, frontal, hacia la derecha, con estandarte y globo.

Exergo: SMNA (Ceca: Nicomedia)

$5 \mathrm{gr} ; 22 \mathrm{~mm} ; 6 \mathrm{~h}$.

Nominal: AE2

Cronología: 379-395 d.C.

Conservación: Buena

\section{$\mathrm{N}^{\circ} 2$}

Anverso: DN ARCADI[VS] PF AV[G]. Busto de Arcadio, diademado, de perfil a la derecha, con coraza. Reverso: [GLORIA] ROMANORVM. Arcadio de pie, frontal, hacia la derecha, con estandarte y globo.

Exergo: SMNB (Ceca: Nicomedia)

$3,73 \mathrm{gr} ; 22 \mathrm{~mm} ; 12 \mathrm{~h}$

Nominal: AE2

Cronología: 383-395 d.C.

Conservación: Regular

\section{$\mathrm{N}^{\circ} 3$}

Anverso: DN ARCADIVS PF AVG. Busto de Arcadio, diademado, de perfil a la derecha, con coraza. Reverso: GLORIA ROMANORVM. Arcadio de pie, frontal, hacia la derecha, con estandarte y globo.

Exergo: SMKA (Ceca: Cirico)

4,40 gr; $21 \mathrm{~mm} ; 7 \mathrm{~h}$.

Nominal: AE2

Cronología: 383-395 d.C.

Conservación: Buena

\section{$\mathrm{N}^{\circ} 4$}

Anverso: DN ARCADIVS PF AVG. Busto de Arcadio, diademado, de perfil a la derecha, con coraza.

Reverso: GLORIA ROMANORVM. Arcadio de pie, frontal, hacia la derecha, con estandarte y globo.

Exergo: SMKB (Ceca: Cirico)

$3,94 \mathrm{gr} ; 22 \mathrm{~mm} ; 12 \mathrm{~h}$.

Nominal: AE2

Cronología: 383-395 d.C.

Conservación: Buena

\section{$\mathrm{N}^{\circ} 5$}

Anverso: DN A[RCADIVS PF AVG]. Busto de Arcadio, diademado, de perfil a la derecha, con coraza.

Reverso: GLORIA ROMANORVM. Arcadio de pie, frontal, hacia la derecha, con estandarte y globo.

Exergo: CONSA (Ceca: Constantinopla)

$3,76 \mathrm{gr} ; 21 \mathrm{~mm} ; 6 \mathrm{~h}$.

Nominal: AE2

Cronología: 383-395 d.C.

Conservación: Regular

\section{$\mathrm{N}^{\circ} 6$}

Anverso: [DN GRATIANVS PF AVG]. Busto de Graciano, diademado, de perfil a la derecha, con coraza.

Reverso: [REPARATIO REIPVB]. Graciano de pie, frontal, hacia la izquierda, con Victoria en la mano izquierda y figura arrodillada representando a los sometidos.

Exergo: No se puede leer.

4,49 gr; $22 \mathrm{~mm} ; 12 \mathrm{~h}$.

Nominal: AE2

Cronología: 367-383 d.C.

Conservación: Mala

\section{$\mathrm{N}^{\circ} 7$}

Anverso: [DN] HONOR[IVS PF AVG]. Busto de Honorio, diademado, de perfil a la derecha, con coraza. Reverso: [GLORIA] ROMANORVM.. Honorio de pie, frontal, hacia la derecha, con estandarte y globo. Exergo: SMNA (Ceca: Nicomedia)

2,63 gr; $19 \mathrm{~mm} ; 12 \mathrm{~h}$.

Nominal: AE2

Cronología: 393-395 d.C.

Conservación: Mala

\section{$\mathrm{N}^{\circ} 8$}

Anverso: [DN THE]ODOSIVS PF AVG. Busto de Teodosio, diademado, de perfil a la derecha, con coraza. Reverso: GLORIA ROMANORVM. Teodosio de pie, frontal, hacia la derecha, con estandarte y globo.

Exergo: SMNA (ceca: Nicomedia)

5,64 gr; $22 \mathrm{~mm}$; I I h.

Nominal: AE2

Cronología: 379-395 d.C.

Conservación: Regular

\section{$\mathrm{N}^{\circ} 9$}

Anverso: DN ARCADIVS PF AVG. Busto de Arcadio, diademado, de perfil a la derecha, con coraza.

Reverso: GLORIA ROMANORVM. Arcadio de pie, frontal, hacia la derecha, con estandarte y globo. Exergo: SM[KA] o SM[NA] (Ceca: Cirico o Nicomedia) 3,59 gr; $22 \mathrm{~mm} ; 6 \mathrm{~h}$.

Nominal: AE2

Cronología: 383-395 d.C.

Conservación: Buena

\section{$\mathrm{N}^{\circ} 10$}

Anverso: [DN HON]O[RIVS PF AVG]. Busto de Honorio, diademado, de perfil a la derecha, con coraza. Reverso: GLORIA RO[MANORVM]. Honorio de pie, frontal, hacia la derecha, con estandarte y globo. Exergo: STR (Ceca: Treveris)

2,29 gr; $21 \mathrm{~mm}$; I h.

Nominal AE2

Cronología: 393-395 d.C.

Conservación: Mala

\section{$\mathrm{N}^{\circ}$ II}

Anverso: [DN ARCA]DIVS PF AVG. Busto de Arcadio, diademado, de perfil a la derecha, con coraza. Reverso: [GL]ORIA ROMANORVM. Arcadio de pie, frontal, hacia la derecha, con estandarte y globo.

Exergo: ANT (Ceca: Antioquía)

$3,95 \mathrm{gr} ; 20 \mathrm{~mm} ; 5 \mathrm{~h}$.

Nominal: AE2

Cronología: 383-395 d.C.

Conservación: Regular 


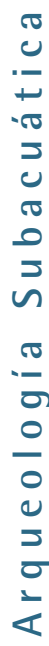

$\mathrm{N}^{\circ} 12$

Anverso: DN THEODOSIVS PF AVG. Busto de Teodosio, diademado, de perfil a la derecha, con coraza. Reverso: GLORIA ROMANORVM. Teodosio de pie, frontal, hacia la derecha, con estandarte y globo.

Exergo: ANTA (Ceca: Antioquía).

3,60 gr; $22 \mathrm{~mm}$; I lh.

Nominal AE2

Cronología: 379-395

Conservación: Buena

\section{$\mathrm{N}^{\circ} 13$}

Anverso: DN VALENTINIANVS PF AVG. Busto de Valentiniano II, diademado, de perfil a la derecha, con coraza. (foto 2)

Reverso: REPARATIO REIPVB. Valentiniano de pie, frontal, hacia la izquierda, con Victoria en la mano izquierda y figura femenina arrodillada.

Exergo: SMRQ (Ceca: Roma)

4,62 gr; $23 \mathrm{~mm}$; $5 \mathrm{~h}$.

Nominal: AE2

Cronología: 375-392 d.C.

Conservación: Buena

\section{$N^{\circ} 14$}

Anverso: DN ARC[ADIVS PF AVG]. Busto de Arcadio, diademado, de perfil a la derecha, con coraza. Reverso: [GLO]RIA ROMANORVM. Arcadio de pie, frontal, hacia la derecha, con estandarte y globo.

Exergo: SMKA (Ceca: Cirico)

3,86 gr; $20 \mathrm{~mm}$; I h.

Nominal: AE2

Cronología: 383-395 d.C.

Conservación: Regular

\section{$\mathrm{N}^{\circ} \mathrm{I5}$}

Anverso: DN THEODO[SIVS PF AVG]. Busto de Teodosio diademado, de perfil a la derecha, con coraza. Reverso: GLO[RIA] ROMANORVM. Teodosio de pie, frontal, hacia la derecha, con estandarte y globo.

Exergo: SMKA (Ceca: Cirico)

$4,10 \mathrm{gr} ; 19 \mathrm{~mm} ; 12 \mathrm{~h}$

Nominal: AE2

Cronología: 379-395 d.C.

Conservación: Regular

\section{$\mathrm{N}^{\circ} 16$}

Anverso: DN VAL[ENTINI]ANVS [PF] AVG. Busto de Valentiniano II diademado, de perfil a la derecha, con coraza.

Reverso: REPARATIO [REIPVB]. Valentiniano de pie, frontal, hacia la izquierda, con Victoria en la mano izquierda,y figura femenina arrodillada.

Exergo: No se puede leer.

$3,12 \mathrm{gr} ; 21 \mathrm{~mm} ; 6 \mathrm{~h}$.

Nominal: AE2

Cronología: 375-392 d.C.

Conservación: Regular

\section{N $\mathrm{N}^{\circ} 17$}

Anverso: DN T[HEODOSIVS PF AVG]. Busto de Teodosio diademado, de perfil a la derecha, con coraza. Reverso: [GLORIA ROMANORVM]. Teodosio de pie, frontal, hacia la derecha, con estandarte y globo.
Exergo: No se puede leer.

$3,30 \mathrm{gr} ; 19 \mathrm{~mm} ; 12 \mathrm{~h}$.

Nominal: AE2

Cronología: 379-395 d.C.

Conservación: Mala

\section{$\mathrm{N}^{\circ} 18$}

Anverso: [DN THEODOSIVS PF AVG]. Busto de Teodosio, diademado, de perfil a la derecha, con coraza. Reverso: [GL]ORIA ROMANORVM. Teodosio de pie, frontal, hacia la derecha, con estandarte y globo.. En el campo estrella.

Exergo: SMHA (Ceca: Heraclea)

4,70 gr; $20 \mathrm{~mm}$; II h.

Nominal: AE2

Cronología: 379-395 d.C.

Conservación: Mala

\section{$N^{\circ} 19$}

Anverso: DN HONORIVS [PF AVG]. Busto de Honorio diademado, de perfil a la derecha, con coraza.

Reverso: GLORIA [ROMANORVM]. Honorio de pie, frontal, hacia la derecha, con estandarte y globo.

Exergo: No se puede leer.

$3,94 \mathrm{gr} ; 20 \mathrm{~mm} ; 5 \mathrm{~h}$.

Nominal: AE2

Cronología: 393-395 d.C.

Conservación: Mala

\section{$\mathrm{N}^{\circ} 20$}

Anverso: DN THEOSOSIVS PF AVG. Busto de Teodosio, diademado, de perfil a la derecha, con coraza. Reverso: GLORIA ROMANORVM. Teodosio de pie, frontal, hacia la derecha, con estandarte y globo. Exergo: [S]MN[A] (Ceca: Nicomedia)

4,67 gr; $21 \mathrm{~mm} ; 5 \mathrm{~h}$.

Nominal: AE2

Cronología: 379-395 d.C.

Conservación: Regular

\section{$\mathrm{N}^{\circ} 21$}

Anverso: DN HO[NORIVS PF AVG]. Busto de Honorio, diademado, de perfil a la derecha, con coraza. Reverso: GL[ORIA] ROMANOR[VM]. Honorio de pie, frontal, hacia la derecha, con estandarte y globo. Exergo: SMNA (Ceca: Nicomedia) $3,83 \mathrm{gr} ; 20 \mathrm{~mm} ; 6 \mathrm{~h}$.

Nominal: AE2

Cronología: 393- 395 d.C.

\section{Conservación}

\section{$\mathrm{N}^{\circ} 22$}

Anverso: DN THEODOSIVS PF AVG. Busto de Teodosio diademado, de perfil a la derecha, con coraza. Reverso: [GL]ORIA ROMANORVM. Teodosio de pie, frontal, hacia la derecha, con estandarte y globo. Exergo: ANTA (Ceca: Antioquía)

4,57 gr; $21 \mathrm{~mm}$; $6 \mathrm{~h}$.

Nominal: AE2

Cronología: 379-395 d.C.

Conservación: Buena

\section{$N^{\circ} 23$}

Anverso: DN THEODOSIVS PF AVG. Busto de Teodosio diademado, de perfil a la derecha, con coraza. 

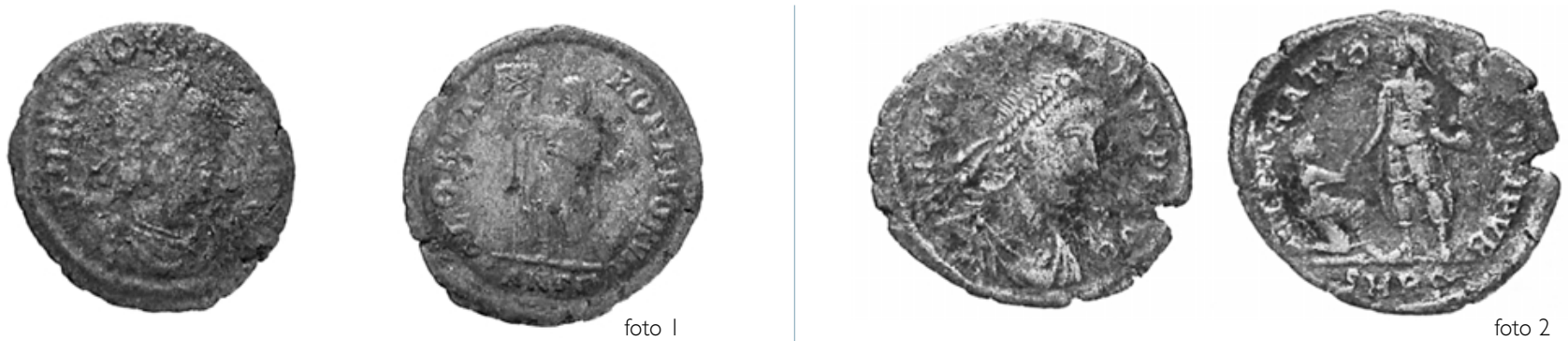

foto 2
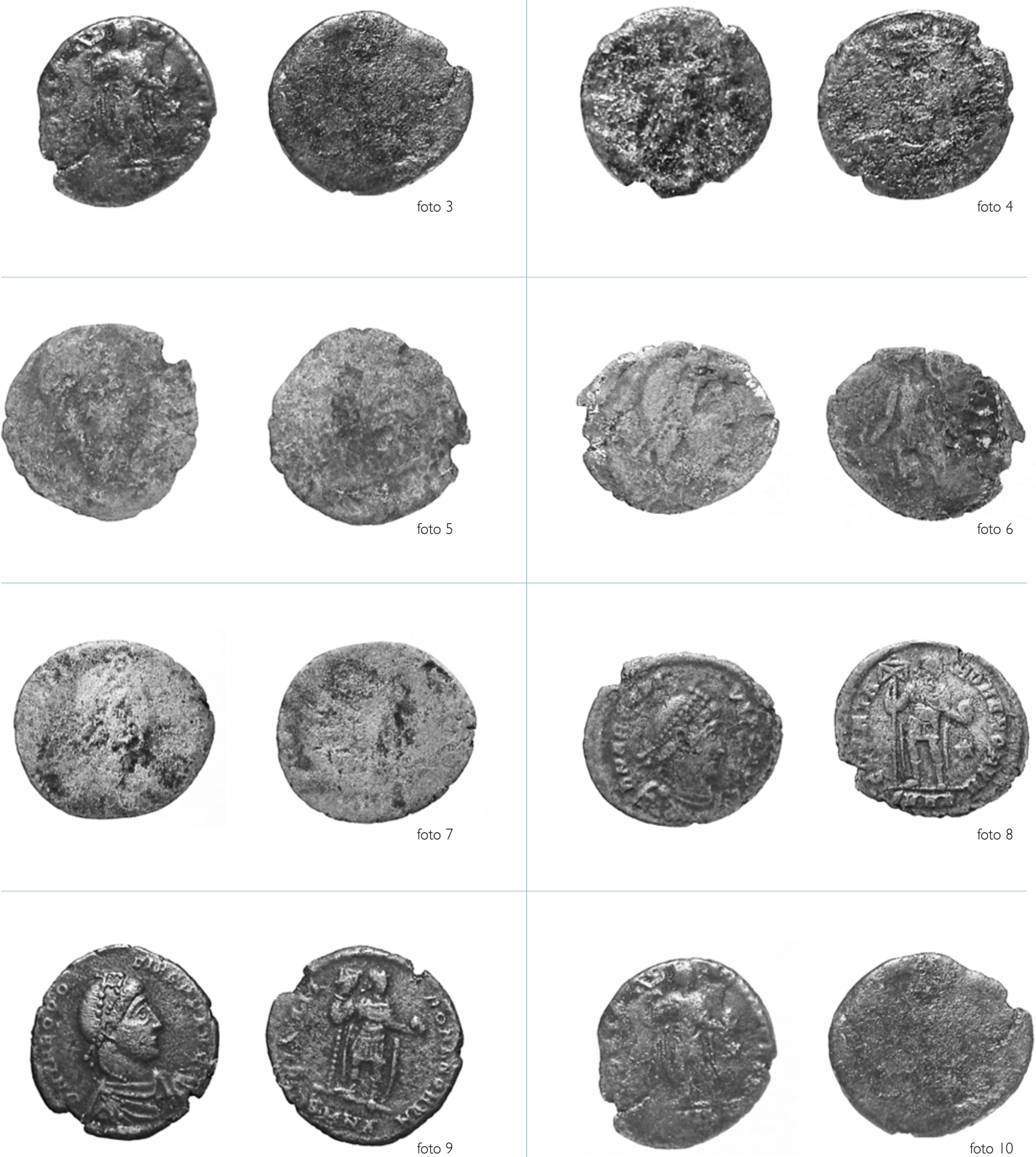
0
0
$+\pi$
2
0
0
0
2
0
0
0
0
0
0
0
2
2
0
1
$<$

Reverso: REPARATIO [REIPVB]. Teodosio de pie, frontal, hacia la izquierda, con Victoria en la mano izquierda, y figura femenina arrodillada.

Exergo: AN[T] (Ceca: Antioquía)

3,64 gr; $22 \mathrm{~mm} ; 6 \mathrm{~h}$.

Nominal AE2

Cronología: 379-395 d.C.

Conservación: Regular

\section{$\mathrm{N}^{\circ} 24$}

Anverso: [DN THEODOSIVS PF AVG]. Busto de Teodosio diademado, de perfil a la derecha, con coraza. Reverso: [REPARATIO REIPVB]. Teodosio de pie, frontal, hacia la izquierda, con Victoria en la mano izquierda, y figura femenina arrodillada.

Exergo: No se puede leer.

$3,63 \mathrm{gr} ; 19 \mathrm{~mm}$; I h.

Nominal: AE2

Cronología: 379-395 d.C.

Conservación: Mala

\section{$\mathrm{N}^{\circ} 25$}

Anverso: [DN GRATIANVS PF AVG]. Busto de Graciano, diademado, de perfil a la derecha, con coraza.

Reverso: [REPARATIO REIPVB]. Graciano de pie, frontal, hacia la izquierda, con Victoria en la mano izquierda, y figura femenina arrodillada.

Exergo: No se puede leer.

$3,52 \mathrm{gr} ; 22 \mathrm{~mm} ; 12 \mathrm{~h}$

Nominal: AE2

Cronología: 367-383 d.C.

Conservación: Mala

\section{$\mathrm{N}^{\circ} 26$}

Anverso: Frustra (foto 3)

Reverso: Emperador de pie, hacia la derecha, con estandarte y globo.

Exergo: SMNA (Ceca: Nicomedia)

4,02 gr; $21 \mathrm{~mm}$

Nominal: AE2

Conservación: Mala

\section{$\mathrm{N}^{\circ} 27$}

Anverso: [DN GRATIANVS PF AVG]. Busto de Graciano diademado, de perfil a la derecha, con coraza. Reverso: [REPA]RATIO [REIPVB]. Graciano de pie, frontal, hacia la izquierda, con Victoria en la mano izquierda, y figura femenina arrodillada.

Exergo: SMRA (Ceca: Roma)

4,49 gr; $22 \mathrm{~mm} ; \mathrm{II}$ h.

Nominal: AE2

Cronología: 367-383 d.C.

Conservación: Mala

\section{$\mathrm{N}^{\circ} 28$}

Anverso: DN THEODO[SIVS PF AVG]. Busto de Teodosio, diademado, de perfil a la derecha, con coraza. Reverso: GLORI[A ROMANORVM]. Teodosio de pie, frontal, a la derecha, con estandarte y globo.

Exergo: ANTA (Ceca: Antioquía).

4,80 gr; $20 \mathrm{~mm}, 6 \mathrm{~h}$.

Nominal: AE2

Cronología: 379-395 d.C.

Conservación: Regular

\section{$N^{\circ} 29$}

Anverso: [DN GRATIANVS PF AVG]. Busto de Graciano diademado, de perfil a la derecha, con coraza. Reverso: GLORIA [ROMANORVM]. Graciano de pie, frontal, a la izquierda, con Victoria en la mano izquierda y figura femenina arrodillada.

Exergo: ANT[A] (Ceca: Antioquía).

2,32 gr; $21 \mathrm{~mm} ; 11 \mathrm{~h}$.

Nominal: AE2

Cronología: 367-383 d.C.

Conservación: Mala

\section{$N^{\circ} 30$}

Anverso: DN HONORIVS PF [AVG]. Busto de Honorio diademado, de perfil a la derecha, con coraza.

Reverso: GLORIA ROMA[NORVM]. Honorio de pie, frontal, hacia la derecha, con estandarte y globo.

Exergo: CONSA (Ceca: Constantinopla).

$3,74 \mathrm{gr} ; 21 \mathrm{~mm} ; 5 \mathrm{~h}$.

Nominal: AE2

Cronología: 393-395 d.C.

Conservación: Regular

\section{$\mathrm{N}^{\circ} 31$}

Anverso: [DN THEODOSIVS PF AVG]. Busto de Teodosio diademado, de perfil a la derecha, con coraza. Reverso: [GLORIA] ROMANORVM. Teodosio de pie, frontal, hacia la derecha, con estandarte y globo. Exergo: CONS (Ceca: Constantinopla)

3,42 gr; $22 \mathrm{~mm} ; 6 \mathrm{~h}$.

Nominal: AE2

Cronología: 379-395 d.C.

Conservación: Mala

\section{$\mathrm{N}^{\circ} 32$}

Anverso: [DN THEODOSIVS PF AVG]. Busto de Teodosio diademado, de perfil a la derecha, con coraza. Reverso: [GLORIA] ROM[ANOR]VM. Teodosio de pie, frontal, hacia la derecha, con estandarte y globo. Exergo: CONS[A] (Ceca: Constantinopla)

$3,36 \mathrm{gr} ; 19 \mathrm{~mm} ; 12 \mathrm{~h}$.

Nominal: AE2

Cronología: 379-395 d.C.

Conservación: Mala

\section{$\mathrm{N}^{\circ} 33$}

Anverso: DN THEODOSIVS PF AVG. Busto de Teodosio diademado, de perfil a la derecha, con coraza. Reverso: GLORIA ROMANORVM. Teodosio de pie, frontal, hacia la derecha, con estandarte y globo.

Exergo: SMNB (Ceca: Nicomedia)

2,85 gr; $21 \mathrm{~mm}$; $6 \mathrm{~h}$.

Nominal: AE2

Cronología: 379-395 d.C.

Conservación: Buena

\section{$\mathrm{N}^{\circ} 34$}

Anverso: DN THEODOSIVS PF AVG. Busto de Teodosio, diademado, de perfil a la derecha, con coraza. Reverso: GLORIA ROMANO[RVM]. Teodosio de pie, frontal, hacia la derecha, con estandarte y globo. Exergo: SMNB (Ceca: Nicomedia)

4,28 gr; $22 \mathrm{~mm} ; 6 \mathrm{~h}$.

Nominal: AE2 
Cronología: 379-395 d.C.

Conservación: Buena

\section{$N^{\circ} 35$}

Anverso: DN T[HEODO]SIVS PF AVG. Busto de Teodosio diademado, de perfil a la derecha, con coraza.

Reverso: REPARAT[IO] REIPVB. Teodosio de pie, frontal, hacia la izquierda, con Victoria en la mano izquierda, y figura femenina arrodillada.

Exergo: SMA[R]P (Ceca: Arlés).

4,65 gr; $21 \mathrm{~mm} ; 2 \mathrm{~h}$.

Nominal: AE2

Cronología: 379-395 d.C.

Conservación: Buena

\section{$\mathrm{N}^{\circ} 36$}

Anverso: [DN THEODOSIVS PF AVG]. Busto de Teodosio diademado, de perfil a la derecha, con coraza. Reverso: [GLORIA ROMANORVM]. Teodosio de pie, frontal, hacia la derecha, con estandarte y globo. Exergo: SMNA (Ceca: Nicomedia).

$3,45 \mathrm{gr} ; 20 \mathrm{~mm} ; 6 \mathrm{~h}$.

Nominal: AE2

Cronología: 379-395 d.C.

Conservación: Mala

\section{$N^{\circ} 37$}

Anverso: DN [THEODOSIVS PF] AVG. Busto de Teodosio diademado, de perfil a la derecha, con coraza.

Reverso: [GLORIA] ROMANORVM. Teodosio de pie, frontal, hacia la derecha, con estandarte y globo. Exergo: SMNA (ceca: Nicomedia).

3, $18 \mathrm{gr} ; 20 \mathrm{~mm} ; 6 \mathrm{~h}$.

Nominal: AE2

Cronología: 379-395 d.C.

Conservación: Regular

\section{$\mathrm{N}^{\circ} 38$}

Anverso: DN ARCADIVS [PF AVG]. Busto de Arcadio diademado, de perfil a la derecha, con coraza. Reverso: GLOR[IA] RO[MANORVM]. Arcadio de pie, frontal, hacia la derecha, con estandarte y globo. Exergo: No se puede leer.

3,29 gr; $20 \mathrm{~mm}$; I h.

Nominal: AE2

Cronología: 383-395 d.C.

Conservación: Regular.

\section{$\mathrm{N}^{\circ} 39$}

Anverso: [DN] ARCAD[IVD PF AVG]. Busto de Arcadio, diademado, de perfil a la derecha, con coraza. Reverso: GLORIA [ROMANORVM]. Arcadio de pie, frontal, hacia la derecha, con estandarte y globo.

Exergo: ALE (Ceca: Alejandría).

3,26 gr; $21 \mathrm{~mm} ; 6 \mathrm{~h}$.

Nominal: AE2

Cronología: 383- 395 d.C.

Conservación: Regular.

\section{$\mathrm{N}^{\circ} 40$}

Anverso: [DN] HONORIVS PF AVG. Busto de Honorio, con diadema de perlas, hacia la derecha, con coraza.
Reverso: GLORIA ROMANORVM. Honorio de pie, frontal, hacia la derecha, con estandarte y globo.

Exergo: ANT (gamma). (Ceca: Antioquía).

$3,77 \mathrm{gr} ; 22 \mathrm{~mm} ; 5 \mathrm{~h}$.

Nominal: AE2

Cronología: 393-395 d.C.

Conservación: Buena.

\section{$\mathrm{N}^{\circ} 4 \mathrm{I}$}

Frustra.

I,82 gr; $17 \mathrm{~mm}$

\section{$\mathrm{N}^{\circ} 42$}

Anverso: DN ARCADIVS [PF] AVG. Busto de Arcadio, diademado, de perfil a la derecha, con coraza. Reverso: GLORIA ROMANORVM. Arcadio de pie, frontal, hacia la derecha, con estandarte y globo.

Exergo: SMNA (Ceca: Nicomedia).

3,8 I gr; $20 \mathrm{~mm} ; 12 \mathrm{~h}$.

Nominal: AE2

Cronología: 383-395 d.C.

Conservación: Buena.

\section{$\mathrm{N}^{\circ} 43$}

Anverso: DN THEODO[SIVS P]F AVG. Busto de Teodosio diademado, a la derecha, con coraza. Reverso: [GL]ORIA [RO]MANOR[VM]. Teodosio de pie, frontal, hacia la derecha, con estandarte y globo. Exergo: No se puede leer.

$3,40 \mathrm{gr} ; 20 \mathrm{~mm} ; 6 \mathrm{~h}$.

Nominal: AE2

Cronología: 379-395 d.C.

Conservación: Regular.

\section{$\mathrm{N}^{\circ} 44$}

Anverso: DN ARCADIVS PF AVG. Busto de Arcadio, diademado, de perfil a la derecha, con coraza. Reverso: GLORIA ROMANORVM. Arcadio de pie, frontal, hacia la derecha, con estandarte y globo.

Exergo: SMNB (Ceca: Nicomedia).

$3,85 \mathrm{gr} ; 21 \mathrm{~mm} ; \mathrm{II} \mathrm{h}$.

Nominal: AE2

Cronología: 379-395 d.C.

Conservación: Buena.

\section{$\mathrm{N}^{\circ} 45$}

Anverso: [DN HO]NORIVS [PF AVG]. Busto de Honorio, diademado, de perfil a la derecha, con coraza. Reverso: GLOR[IA RO]MANORVM. Honorio de pie, frontal, hacia la derecha, con estandarte y globo.

Exergo: ANT (Ceca: Antioquía).

4,34 gr; $21 \mathrm{~mm} ; 5 \mathrm{~h}$.

Nominal: AE2

Cronología: 393-395 d.C.

Conservación: Regular.

\section{$\mathrm{N}^{\circ} 46$}

Anverso: [DN THEO]DOSIVS PF AVG. Busto de Teodosio, diademado, de perfil a la derecha, con coraza. Reverso: GLO[RIA] ROMANORVM. Teodosio de pie, frontal, hacia la derecha, con estandarte y globo. Exergo: ANTA (Ceca: Antioquía).

4,23 gr; $21 \mathrm{~mm} ; 5 \mathrm{~h}$.

Nominal: AE2 
0
0
1
$-\pi$
2
0
0
0
5
0
0
0
0
0
0
0
0
2
0
1
$<$

Cronología: 379-395 d.C.

Conservación: Regular.

\section{$N^{\circ} 47$}

Anverso: Busto de emperador, de perfil, diademado, a la derecha, con coraza. (foto 4)

Reverso: GLO[RIA ROMANORVM]. Emperador de pie, frontal, hacia la derecha, con estandarte y globo.

Exergo: No se puede leer.

$3,46 \mathrm{gr} ; 21 \mathrm{~mm} ; \mathrm{II} \mathrm{h}$.

Nominal: AE2

Cronología: 337-395 d.C.

Conservación: Mala.

\section{$\mathrm{N}^{\circ} 48$}

Anverso: DN THEO[DOSIVS PF] AVG. Busto de Teodosio, diademado, de perfil a la derecha, con coraza.

Reverso: [GLO]RIA ROMANORVM. Teodosio de pie, frontal, hacia la derecha, con estandarte y globo.

Exergo: No se puede leer.

$4,25 \mathrm{gr} ; 19 \mathrm{~mm} ; 6 \mathrm{~h}$.

Nominal: AE2

Cronología: 379-395 d.C.

Conservación: Regular.

\section{$\mathrm{N}^{\circ} 49$}

Anverso: [DN T]HEODOSIVS PF AVG. Busto de Teodosio, diademado, de perfil a la derecha, con coraza. Reverso: GLORIA ROMANORVM. Teodosio de pie, frontal, hacia la derecha, con estandarte y globo.

Exergo: ANTA (Ceca: Antioquía).

3,23 gr; $20 \mathrm{~mm} ; 5 \mathrm{~h}$.

Nominal: AE2

Cronología: 379-395 d.C.

Conservación: Regular.

\section{$\mathrm{N}^{\circ} 50$}

Anverso: DN [VALENTIN]IANVS PF AVG. Busto de Valentiniano II, diademado, de perfil a la derecha, con coraza.

Reverso: [REPARA]TIO REIPVB. Valentiniano de pie, frontal, hacia la izquierda, con Victoria en la mano izquierda, y figura femenina arrodillada.

Exergo: SMNA.(Ceca: Nicomedia).

4,8I gr; $22 \mathrm{~mm} ; 6 \mathrm{~h}$.

Nominal: AE2

Cronología: 375-292 d.C.

Conservación: Regular.

\section{$\mathrm{N}^{\circ} 5 \mathrm{I}$}

Anverso: DN HONORIVS [PF AVG]. Busto de Honorio, diademado, de perfil a la derecha, con coraza. Reverso: GLORIA [ROMANO]RVM. Honorio de pie, frontal, hacia la derecha, con estandarte y globo.

Exergo: SMN (Ceca: Nicomedia)

3,85 gr; $21 \mathrm{~mm} ; 6 \mathrm{~h}$.

Nominal: AE2

Cronología: 393-395 d.C.

Conservación: Regular.

$\mathrm{N}^{\circ} 52$

Anverso: DN ARCADIVS PF AVG. Busto de Arcadio, diademado, de perfil a la derecha, con coraza.
Reverso: GLORIA ROMANORVM. Arcadio de pie, frontal, hacia la derecha, con estandarte y globo.

Exergo: SMKA (Ceca: Cirico)

5,33 gr; $20 \mathrm{~mm} ; 7 \mathrm{~h}$.

Nominal: AE2

Cronología: 383-395 d.C.

Conservación: Buena

\section{$\mathrm{N}^{\circ} 54$}

Anverso: DN AR[CADIVS] PF AVG. Busto de Arcadio, diademado, de perfil a la derecha, con coraza.

Reverso: [GLORIA] ROMANORVM. Arcadio de pie, frontal, hacia la derecha, con estandarte y globo.

Exergo: CONST (Ceca: Constantinopla).

$3,93 \mathrm{gr} ; 20 \mathrm{~mm} ; 12 \mathrm{~h}$.

Nominal: AE2

Cronología: 383-395 d.C.

Conservación: Regular.

\section{$\mathrm{N}^{\circ} 55$}

Anverso: [DN MAG MAXIMVS PF]. Busto de Magno Máximo, diademado, de perfil a la derecha, con coraza. Reverso: [REPARATIO REIPVB]. Magno Máximo de pie, hacia la izquierda, con figura femenina arrodillada. Exergo: No se puede leer.

4,46 gr; $22 \mathrm{~mm} ; 12 \mathrm{~h}$.

Nominal: AE2

Cronología: 383-388 d.C.

Conservación: Mala

\section{$\mathrm{N}^{\circ} 56(1 / 99)$}

Anverso: [DN CONSTANS PF AVG]. Busto de Constante, diademado, de perfil a la derecha, con coraza. (foto 5)

Reverso: [FEL.TEMPREPARATIO]. Constante de pie, sobre una galera, con estandarte en una mano y ave en la otra.

Exergo: TR [-](Ceca: Treveris).

I,2I gr; $15 \mathrm{~mm} ; 12 \mathrm{~h}$.

Nominal: AE4

Cronología: 346-350 d.C.

Conservación: Mala

\section{$\mathrm{N}^{\circ} 57(3 / 99)$}

Anverso: [DN MAG MAXIMVS PF AVG]. Busto de Magno Máximo, diademado, de perfil a la derecha, con coraza. (foto 6 )

Reverso: [REPARATIO REIPVB]. Magno Máximo de pie, hacia la izquierda, con figura femenina arrodillada. Exergo: No se puede leer.

2,72 gr; $22 \mathrm{~mm} ; 6 \mathrm{~h}$.

Nominal: AE2

Cronología: 383-388 d.C.

Conservación: Mala

\section{$\mathrm{N}^{\circ} 58(4 / 99)$}

Anverso: [DN GRATIANVS PF AVG]. Busto de Graciano, diademado, de perfil a la derecha, con coraza. (foto 7 )

Reverso: [REPARATIO REIPVB]. Graciano de pie, frontal, hacia la derecha, con estandarte y globo.

Exergo: SMHA (Ceca: Heracleia).

5,02 gr; $21 \mathrm{~mm}$; I h.

Nominal: AE2 
Cronología: 367-383 d.C.

Conservación: Mala

$N^{\circ} 59(6 / 99)$

Anverso: DN ARCAD[IVS PF AVG]. Busto de Arcadio, diademado, de perfil a la derecha, con coraza. Reverso: GLORIA ROMANORVM. Arcadio de pie, frontal, hacia la derecha, con estandarte y globo. Exergo: SMNB (Ceca: Nicomedia).

4, $12 \mathrm{gr} ; 22 \mathrm{~mm} ; 12 \mathrm{~h}$.

Nominal: AE2

Cronología: 383-395 d.C.

Conservación: Regular

\section{$\mathrm{N}^{\circ} 60(8 / 99)$}

Anverso: DN ARCADI[VS PF] AVG. Busto de Arcadio, diademado, de perfil a la derecha, con coraza. (foto 8)

Reverso: GLORIA ROMANORVM. Arcadio de pie, frontal, hacia la derecha, con estandarte y globo.
Exergo: SMNB (Ceca: Nicomedia). Estrella en el campo. 4,67 gr; $22 \mathrm{~mm} ; 12 \mathrm{~h}$.

Nominal: AE2

Cronología: 383-395 d.C.

Conservación: Buena

\section{$\mathrm{N}^{\circ} 6 \mathrm{I}(8 \mathrm{a} / 99)$}

Anverso: [DN] HONORIVS [PF AVG]. Busto de Honorio, diademado, de perfil a la derecha, con coraza. (foto 9)

Reverso: GLORIA ROMANORVM. Honorio de pie, frontal, hacia la derecha, con estandarte y globo.

Exergo: ANT (Ceca: Antioquía).

$5,25 \mathrm{gr} ; 22 \mathrm{~mm} ; 12 \mathrm{~h}$.

Nominal: AE2

Cronología: 393-395 d.C.

Conservación: Buena

\section{Bibliografía}

ABAD VARELA, M. (1989), Circulación monetaria en la Hispania romana del siglo IV J.C, (microfichas), Madrid.

IDEM. (|99|), "Algunas cuestiones sobre las tesaurizaciones durante el siglo IV d.C. en Hispania", Actas VII Congreso Nacional de Numismática, Madrid, p.235-252.

IDEM. (1994), "Circulación monetaria durante el Bajo Imperio romano", Actas VIII Congreso Nacional de Numismática (Avilés, 1992), Madrid, p. |49-166.

IDEM. (1995), "Depósito monetario del siglo IV d.C. Procedente del Bierzo (León)", Actas del IX Congreso Nacional de Numismática (Elche 1994), Alicante, p. 263-278.

ALFARO ASINS, C. (I99|), "Tesoro de solidos hallado en Arcos de la Frontera (Cádiz)", Actas del VII Congreso Nacional de Numismática, Madrid, p. 393-408.

DEPEYROT, G. (1987), Le Bas Empire Romain. Economie et numismatique (284-49I), París.

GALLARDO, M.; MARTÍ, J.; ALONSO, C.; GARCÍA C. (1994) "Prospecciones arqueológicas subacuáticas en Sancti-Petri". Anuario Arqueológico de Andalucía, Tomo II, Sevilla, p. 44-48
GALLARDO, M.; MARTÍ, J.; ALONSO, C; GARCÍA, C. (1995) "Prospecciones arqueológicas subacuáticas en Sancti-Petri. Proyecto general de investigación de la Bahía de Cádiz". Anuario Arqueológico de Andalucía, Tomo II, Sevilla, p. I5-25

GALLARDO, M.; MARTÍ, J.; ALONSO, C; GARCÍA, C. (1995) "Carta arqueológica subacuática de la Bahía de Cádiz". Cuadernos de Arqueología Marítima, 3. Cartagena, p. 105- 122

HILL, P.V.; CARSON, R.A.G.; KENT, J.P.C. (1978), Late Roman Bronze Coinage, N. York

KENT, J.P.C. (1994), The Roman Imperial Coinage, (ed. Por R.A.G. Carson, J.P.C. Kent y A.M. Burnett), vol. X, Londres

NONY, D. (1967), "Un trésor monétaire du Bas-Empire à Tarifa (Cádiz), Mélanges de la Casa de Velázquez III, p. 94-I I4.

PÉREZ SINDREU, F. (1982), "Tesorillo de monedas del bajo Imperio encontrado en La Lentejuela (Sevilla)", Numisma, p. 91 - I I0.

\section{Nota}

I. Proyecto aprobado por la Dirección General de Bienes Culturales en el año 1992, bajo la coordinación de Mercedes Gallardo Abárzuza y la dirección de Josefa Martí Solano, Carmen García Rivera y Carlos Alonso Villalobos. 